\title{
Armut und soziale Netzwerke: Empirische Befunde
}

Bereits Jahoda et al. (1975 [1933]) untersuchten in ihrer wegweisenden Studie für die qualitative und methodenintegrative Sozialforschung die Auswirkungen der nahezu flächendeckenden Erwerbslosigkeit auf die sozialen Beziehungen der Bewohner*innen von Marienthal, einem österreichischen Fabrikdorf mit 478 Haushalten, dem die Fabrik abhandengekommen war. Die Folgen der Erwerbslosigkeit beschreiben die Autor*innen so:

„Die Ansprüche an das Leben werden immer weiter zurückgeschraubt; der Kreis der Dinge und Einrichtungen, an denen noch Anteil genommen wird, schränkt sich immer mehr ein; die Energie, die noch bleibt, wird auf die Aufrechterhaltung des immer kleiner werdenden Lebensraumes konzentriert. " (ebd. S. 101)

Aus der im vorigen Kapitel eingeführten theoretischen Perspektive der Netzwerkforschung betrachtet, bedeutet das einen schrittweisen Wegfall von Domänen. Dazu zählen der Arbeitsplatz, die Mitgliedschaft und Partizipation in Vereinen und Parteien, der Besuch kultureller Veranstaltungen oder die Nutzung der Bibliothek, kurz: das Interesse an jeglichen Aktivitäten, die über die absolut notwendigen Dinge hinausgehen (ebd., insb. S. 55-63). Für dieses Phänomen des „Einschrumpfen[s] von Lebensäußerungen“ (ebd., S. 57) führen die Autor*innen drei wesentliche Gründe an:

1. materielle Knappheit und die Notwendigkeit einer sparsamen Lebensführung,

2. eine charakteristische Grundhaltung der Befragten (ein Interviewpartner sagt z. B.: „Man hat den Kopf nicht danach [...] Mein Gott, man hat jetzt andere Sorgen“, ebd., S. 58.), sowie

3. eine Veränderung der Bedeutung von Zeit, die im Überfluss vorhanden zu sein scheint und daher jegliche Eile obsolet macht. 
Auch wenn diese Erkenntnisse aus heutiger Sicht teilweise etwas überspitzt formuliert und zu stark auf die Gruppe der Arbeitslosen insgesamt verallgemeinert wirken, ist die Studie wegweisend, da sie Erwerbslosigkeit und Armut als ein subjektives Phänomen rekonstruiert, welches im Kontext makro- und vor allem mesostruktureller Einbettungen erfahren wird. Diese Perspektive war auch leitend bei der im Folgenden zusammengefassten Literaturrecherche. Wie sich zeigen wird, werden die grundlegenden Ergebnisse von Jahoda et al. (1975 [1933]) bis heute immer wieder reproduziert und vertieft.

Eine Vorbemerkung scheint noch sinnvoll: Besonders im Literaturteil dieser Arbeit lässt es sich kaum vermeiden, dass die klare Trennung zwischen den Begriffen (relative) Armut, (Langzeit-)Erwerbslosigkeit und Prekarität verwischt. Armut wird in dieser Arbeit als ein übergeordnetes Konstrukt verstanden, welches die wahrgenommene oder tatsächliche Zugehörigkeit zu einer als angemessenen empfundenen Statusgruppe in den unteren Einkommensquintilen der Gesellschaft bedroht (siehe Abschnitt 2.3). Darunter fallen also auch Phänomene wie Arbeitsplatzunsicherheit, Erwerbslosigkeit und Prekarität, sofern sie den sozialen Status und die Teilhabe der Betroffenen infrage stellen und die materielle Absicherung der alltäglichen Lebensführung gefährden. Viele Studien fokussieren auf einzelne Teilbereiche dieses Phänomens, die für die Betrachtung der Wahrnehmung und Bewältigung von Armut insgesamt jedoch von großem Interesse sind. Wenn die Autor*innen dieser Studien z. B. lediglich über »Erwerbslosigkeit« oder »Prekarität« schreiben, wird diese Wortwahl hier übernommen. In den Schlussfolgerungen wird dann jedoch schrittweise auf den Armutsbegriff abstrahiert.

Die ersten beiden Abschnitte des Literaturteils beschäftigen sich mit den Wechselwirkungen zwischen Armut und sozialen Beziehungsnetzwerken. Armut beeinflusst soziale Netzwerke hinsichtlich ihrer Größe, ihrer Zusammensetzung und der Qualität der Beziehungen, also des Umfangs der Ressourcen, die sich aus Netzwerken ziehen lassen (3.1). In der entgegengesetzten Richtung werden Mechanismen des sozialen Drucks (z. B. in Peergruppen) sowie ressourcenintensiver Anstrengungen der Anpassung an statushöhere Gruppen (Armut verbergen, versuchen, ,oben“ mitzuhalten) diskutiert, die dazu führen können, dass sich die Armutssituation reproduziert oder sogar verschärft (3.2). Im dritten Abschnitt werden Typologien betrachtet, die diese Zusammenhänge innerhalb der Gruppe der Armen differenziert betrachten (3.3). Schließlich werden die Ergebnisse des Literaturstudiums in einem zusammenfassenden Abschnitt reflektiert, um daraus Überlegungen und Fragestellungen im Hinblick auf die eigene empirische Untersuchung abzuleiten (3.4). 


\subsection{Wie wirkt Armut auf soziale Netzwerke?}

Die Auswirkungen von Armut auf soziale Netzwerke lassen sich in drei wesentliche Zusammenhänge einordnen, die sich vor allem aus quantitativen Längsschnittanalysen und biographisch rekonstruktiven Betrachtungen ableiten lassen, die jedoch bislang noch relativ selten sind (z. B.: Böhnke \& Link, 2017, 2018):

1. Netzwerkgröße: Viele Netzwerke von Menschen in Armut schrumpfen mit der Zeit an ihren Rändern - insbesondere schwache Beziehungen zu Freund*innen und Kolleg*innen werden mit andauernder Armut weniger, während ein Kern aus starken Beziehungen zu engen Freund*innen und Familienmitgliedern erhalten bleibt.

2. Netzwerkzusammensetzung: In der Folge kommt es zu einer Veränderung der Netzwerkzusammensetzung - der Anteil familiärer Beziehungen steigt, der Anteil statushöherer Gruppen (z. B. Erwerbstätige in den Netzwerken erwerbsloser Personen) sinkt mit der Zeit.

3. Netzwerkqualität und -ressourcen: Die Beziehungsintensität zu Personen außerhalb des Kernnetzwerks und die im Netzwerk verfügbare Unterstützung nimmt mit zunehmender Dauer der Armut ab.

Im Folgenden werden die drei Mechanismen im Detail beschrieben und Erklärungsansätze dazu erläutert.

\subsubsection{Einflüsse auf die Netzwerkgröße}

Armut führt häufig dazu, dass sich die Zahl der Personen, mit denen die Betroffenen im Alltag interagieren, reduziert. Als ursächlich für den Ausschluss oder Rückzug aus solchen Verkehrskreisen werden in der empirischen Literatur folgende Gründe genannt:

1. Formaler Ausschluss, z. B. durch Kündigung der Arbeitsstelle (Grimm et al., 2013; Klärner \& Knabe, 2016; Kreher \& Matthäus, 2012; Kutzner, 2016; Marquardsen, 2012; Weißmann, 2016).

2. Stigmatisierung und Diskriminierung können dazu führen, dass von Armut betroffene Menschen sich z. B. aus Scham aus Beziehungen zurückziehen (Gurr \& Lang, 2018; Knabe, Fischer et al., 2018; Krug et al., 2019). 
3. Tatsächliche oder durch die Betroffenen angenommene Reziprozitätsnormen können zum Verlust von Beziehungen führen, wenn diese infolge der Armut nicht mehr erfüllt werden können. Betroffene ziehen sich dann zurück oder werden aufgrund »offener Rechnungen « aus Beziehungen ausgeschlossen (Gefken, 2018; Marquardsen, 2012; Nelson, 2000; Noordhoff, 2008; Offer, 2012; Phan et al., 2009).

4. Ein Mangel an Gelegenheitsstrukturen in stärker von Armut betroffenen Nachbarschaften kann dazu führen, dass es Personen in ärmeren Gegenden schwerer fällt, soziale Beziehungen einzugehen und zu pflegen (Cattell, 2001; Cornwell \& Behler, 2015; Domínguez \& Watkins, 2003; Friedrichs \& Oberwittler, 2007; Klärner \& Knabe, 2019; Petermann, 2015).

5. Überforderung mit der Pflege sozialer Beziehungen aufgrund erhöhter Anforderungen an die Alltagsbewältigung unter den Bedingungen von Armut und Prekarität (Andresen \& Galic, 2015; Gefken, 2018; Grimm et al., 2013; Ryser \& Halseth, 2011).

Da die Wirksamkeit und Relevanz dieser Mechanismen in der Regel von konkreten Akteurskonstellationen und subjektiven Wahrnehmungsweisen abhängen, lassen sie sich nicht universal auf alle Erscheinungsformen von Armut anwenden. Eine eingehendere Betrachtung dieser fünf Formen der Auswirkungen von Armut auf Netzwerke ist daher nötig.

\section{1.) Formaler Ausschluss}

Viele Studien weisen darauf hin, dass die Erwerbsarbeit neben der Familie die wichtigste Referenz bei der Wahrnehmung sozialer und gesellschaftlicher Teilhabe darstellt - selbst dann, wenn sie prekär oder ganz unterbrochen wird (Grimm et al., 2013; z. B.: Kreher \& Matthäus, 2012; Kutzner, 2016). Insofern bedeutet der Ausschluss von Erwerbsarbeit nicht nur den Wegfall vieler kontextgebundener Beziehungen auf der Arbeitsstelle, sondern darüber hinaus auch eine Infragestellung der sozialen Stellung der Betroffenen in weiteren Domänen ihrer Netzwerke. Dieser materiellen und sozialen Verunsicherung muss durch ein kompensatorisches Handeln begegnet werden (Klärner \& Knabe, 2016), mithilfe dessen die soziale Zugehörigkeit in einigen Fällen wiederhergestellt werden kann (Marquardsen, 2012; Weißmann, 2016 - siehe unten).

\section{2.) Stigmatisierung und Diskriminierung}

Menschen in Armut müssen sich vieler negativer Zuschreibungen erwehren, die in vielen Diskursen immer wieder auftreten (Medien, Politik, Wirtschaft, etc.). 
Die subjektiven Strategien im Umgang mit diesen Zuschreibungen haben auch Auswirkungen auf die Netzwerkgröße.

Gurr und Lang (2018) zeigen, dass insbesondere Erwerbslose mit Deprivationserfahrungen und solche, die wiederkehrend oder über einen längeren Zeitraum erwerbslos sind, dazu neigen, sich aus Beziehungen zu erwerbstätigen Personen zurückzuziehen oder Stigmatisierungen zu vermeiden, indem sie z. B. versuchen, sich aus ,gemischte[n] Interaktionssituationen“ (ebd. S. 268) mit Nicht-Erwerbslosen herauszuhalten.

Auch die Chancen auf (Re-)Integration in Arbeit werden durch Stigmatisierung negativ beeinflusst. Krug et al. (2019) machen dies anhand eines tragischen Analyseergebnisses deutlich. Auf Basis einer quantitativen Längsschnittanalyse des PASS-Datensatzes zeigen sie, dass Erwerbslose, die sich einer negativen Stigmatisierung von »Arbeitslosigkeit « bewusst sind, mehr Anstrengungen unternehmen, um wieder Arbeit zu finden. Gleichzeitig sind ihre subjektiven Erwartungen an einen Erfolg ihrer Bemühungen geringer - und tatsächlich lässt sich kein positiver Effekt ihrer verstärkten Aktivitäten auf die (Re-)Integration in den Arbeitsmarkt feststellen. Demzufolge können Langzeitarbeitslose ihre Diskriminierung auf dem Arbeitsmarkt auch dann nicht verringern, wenn sie sich den über die Stigmatisierung an sie herangetragenen normativen Anforderungen unterwerfen. Die mit der Erwerbsarbeit verbundenen Domänen bleiben ihnen dauerhaft verschlossen.

Knabe et al. (2018) zeigen, dass von Armut betroffene Menschen versuchen, Domänen, in denen sie dem Risiko der Stigmatisierung aufgrund ihrer sozialen Lage ausgesetzt sind, entweder ganz zu meiden oder dass sie sich dort so verhalten, dass ihre Armut nicht zum Thema wird. Aufenthalte in statushöheren Gruppen werden dadurch kostspieliger und seltener oder unterbleiben ganz.

\section{3.) Ausgrenzende Reziprozität}

Offer (2012) systematisiert empirische Befunde ethnologischer Studien zur Bedeutung von Reziprozitätserwartungen in Bezug auf die Verfügbarkeit sozialer Unterstützung in den Netzwerken von Armut betroffener Personen. Sie stellt fest, dass Reziprozität hier nicht - wie allgemein angenommen - als sozialer Kitt der Gesellschaft verstanden werden kann, sondern häufig zu Rückzug und Exklusion führt. Personen in Armut werden einerseits aus Netzwerken ausgeschlossen, weil sie deren Ressourcen einseitig nutzen, ohne etwas zurückzugeben (materielle Dimension) oder weil sie den Status der anderen Netzwerkmitglieder gefährden (normative Dimension). Andererseits ziehen sie sich selbst aus Beziehungen zurück, weil sie die Ressourcen der anderen nicht einseitig ausnutzen 
wollen (materielle Dimension) oder weil sie sich der Kritik der anderen an ihrer Bedürftigkeit entziehen möchten (normative Dimension).

Auf Basis einer qualitativen Studie mit 39 alleinerziehenden Müttern mit geringem Einkommen aus dem ländlichen Raum im US-Bundesstaat Vermont stellt Nelson (2000) fest, dass Reziprozität auch dann besonders wichtig ist, wenn der eigene Unterstützungsbedarf sehr hoch ist. Das liegt einerseits an der Wirksamkeit einer allgemeinen Reziprozitätsnorm, andererseits an dem Bedürfnis der befragten Mütter nach Unabhängigkeit und Selbstbestimmung, welches zur Vermeidung einseitiger Unterstützungsverhältnisse führt.

Gefken (2018) weist darauf hin, dass die Pflege und Aufrechterhaltung von Freundschaftsbeziehungen in prekären Lebenslagen in besonderem Maße von der Verlässlichkeit und Verfügbarkeit sozialer Unterstützung aus der Herkunftsfamilie abhängt. Wenn Freunde einseitig materielle Unterstützung leisten, wird die Reziprozitätsnorm verletzt und die Freundschaften prekär. Federt die Herkunftsfamilie materielle Notlagen ab, geraten Freundschaften weniger unter Druck (ebd.). Die in den Domänen sozialer Netzwerke ablaufenden Mechanismen sollten daher nicht isoliert voneinander betrachtet werden.

\section{4.) Bedeutung von Gelegenheitsstrukturen}

Besonders eindrücklich verweist Small (2009) auf die Bedeutung von Kontexten und Gelegenheitsstrukturen für die Entstehung von unterstützenden sozialen Beziehungen. In seiner vergleichenden Analyse der Netzwerke von Müttern arbeitet er heraus, dass die Aktivitäten von Einrichtungen zur Förderung des Austauschs mit Erzieher*innen und anderen Eltern sogar bedeutsamer für die Entstehung von Unterstützungsnetzwerken sein können als die individuellen Strategien des Netzwerkhandelns der Befragten.

Petermann (2015) betont die Bedeutung von Nachbarschaften als Gelegenheitsstrukturen zur Entstehung und Pflege sozialer Beziehungen. So können zufällige Begegnungen in öffentlichen Räumen oder bei gemeinsamen Aktivitäten in Wohnortnähe zur Entstehung von Beziehungen jenseits institutioneller Eingebundenheit beitragen.

Die Verfügbarkeit dieser Gelegenheitsstrukturen unterscheidet sich jedoch je nach Ausstattung der Nachbarschaften mit kulturellen Einrichtungen, öffentlichen Orten, Cafés und Ähnlichem, wie Cornwell und Behler (2015) zeigen. Sie beobachten in diesem Zusammenhang in den USA, dass die Netzwerke älterer Menschen in benachteiligten Nachbarschaften (gemessen an Indikatoren wie Erwerbslosigkeit, Anteil an Personen ohne Schulabschluss, Anteil alleinerziehender Frauen, Armutsqoute usw.) kleiner sind als die von älteren Menschen in »besseren« Gegenden und dass die Beziehungen der männlichen Befragten in 
»unordentlichen « Nachbarschaften ${ }^{1}$ schwächer sind. Insbesondere in stark von Armut und Deprivation betroffenen Nachbarschaften ist die Chance, der Armut durch den Rückgriff auf nachbarschaftliche Ressourcen zu entkommen, gering hier kann die Wohnsituation sogar zur Verstärkung der wahrgenommenen Armut und Chancenlosigkeit führen (Noordhoff, 2008, 129 f.). Im Extremfall kann die strukturelle Benachteiligung einer Region zu selektiver Abwanderung qualifizierter und chancenreicher Bevölkerungsteile führen, die eine Erosion der Netzwerke der verbliebenen Personen zur Folge hat (Klärner \& Knabe, 2019).

\section{5.) Reduktion von Beziehungen aufgrund alltäglicher Belastungen}

Wellman und Potter (1999) zeigen in ihrer Analyse persönlicher Beziehungsnetzwerke, dass diese in der Regel aus einem festen Kern bestehen, der von loseren Beziehungen und Kontexten umgeben ist: „Many community networks might consist of a „saved“ core and a „liberated“ periphery“ (ebd., S. 50, Hervorhebung im Original). Untersuchungen zu Prekarität im Lebensverlauf zeigen, dass von Armut betroffene Menschen häufig nicht mehr die Kraft haben, in die Aufrechterhaltung der Peripherie ihrer Netzwerke zu investieren. Um die erhöhten Anforderungen eines prekären Alltags bewältigen zu können, werden nur noch die wirklich wichtigen Kontakte gepflegt und Beziehungen darüber hinaus fallengelassen (Andresen \& Galic, 2015; Gefken, 2018; Grimm et al., 2013; Ryser \& Halseth, 2011). Diese Strategie kann im schlimmsten Fall zur Überforderung des Kernnetzwerkes bei gleichzeitigem Verlust darüber hinausgehender sozialer und gesellschaftlicher Teilhabe führen (ebd.). So lässt sich ein erhöhtes Scheidungsrisiko unter der Bedingung von Armut und Prekarität feststellen (Hansen, 2005). Auch Jahoda et al. (1975 [1933]) konstatierten einen Rückzug in die familiären Beziehungen und eine damit einhergehende verstärkte Belastung der Ehen, die sich unter den Arbeitslosen von Marienthal jedoch nicht in einer erhöhten Trennungsneigung während des Beobachtungszeitraums wiederspiegelte.

Um zu großen Verallgemeinerungen entgegenzuwirken, muss der hier vorgenommenen Diskussion vorwiegend negativer Einflüsse von Armut auf die Netzwerkgröße noch eine bedeutsame Einschränkung hinzugefügt werden. International vergleichende Studien weisen darauf hin, dass der Zusammenhang zwischen Armut und Netzwerkgröße in Abhängigkeit vom gesellschaftlichen Umgang mit der Armut variiert (Paugam, 2008; Paugam \& Russell, 2000 - ausführlich dazu: siehe Abschnitt 2.1). Böhnke (2008) zeigt, dass die ausgrenzende

${ }^{1}$ „disordered neighborhoods“ ebd., S. 311; gemeint ist eine negative subjektive Einschätzung der Interviewer*innen zu Sauberkeit, Zustand der Gebäude, Verschmutzung, Baufälligkeit am Wohnort der Interviewten. 
Wirkung von Armut in Ländern, in denen Einkommensarmut nur selten vorkommt (z. B. Schweden, Luxemburg, Deutschland), besonders groß ist. Hier sind von Armut betroffene Menschen häufiger von sozialer Desintegration bedroht ${ }^{2}$, während die Armut in Ländern mit relativ vielen Einkommensarmen (z. B. Malta, Spanien und Polen) weniger ausgrenzend wirkt ${ }^{3}$. In eine ähnliche Richtung weisen die Analysen von Letki und Mierina (2015). Sie zeigen, dass die Netzwerke der unteren Schichten in ökonomisch stark segregierten Gesellschaften (wie Russland oder Brasilien) sogar größer sein können als die der oberen Schichten. Sie erklären diesen Zusammenhang damit, dass die Unterstützungspotentiale der statushomogenen Beziehungen in segregierten Gesellschaften für untere Schichten besonders niedrig sind. Daher müssten deren Angehörige sehr viele Beziehungen aufnehmen und pflegen, um ausreichend Unterstützung aus dem Netzwerk zu generieren, während statushohe Personen bereits aus wenigen Beziehungen zu ebenfalls statushohen Akteur*innen ausreichend Unterstützung ziehen ${ }^{4}$.

\subsubsection{Einflüsse auf die Netzwerkzusammensetzung}

Auf den ersten Blick scheint es, dass andauernde Armut zu einer Homogenisierung von Netzwerken führt, die eng mit den oben diskutieren Einflüssen auf die Netzwerkgröße zusammenhängt. Armut erschwert die Aufrechterhaltung und Aufnahme von Beziehungen und führt daher mit der Zeit zu einer Reduktion auf ein Kernnetzwerk aus überwiegend familiären Akteur*innen und Personen in

\footnotetext{
${ }^{2}$ Soziale Desintegration wird hier jedoch nicht unmittelbar über das Netzwerk gemessen, sondern eher indirekt erfasst, durch einen Indikator, der subjektive Einschätzungen wie die Kontakthäufigkeit mit Freunden, fehlende Unterstützung in Notfällen, Unzufriedenheit mit dem sozialen bzw. dem Familienleben sowie die Wahrnehmung unzureichender sozialer Integration zusammenfasst.

${ }^{3}$ Dass die Sensibilität für kulturelle Unterschiede auch innerhalb von Armutspopulationen in reichen Ländern wie Norwegen bedeutsam sein kann, zeigen die überraschenden Befunde von Dahl et al. (2008). Sie finden einen positiven bivariaten Effekt relativer Einkommensarmut auf die Kontakthäufigkeit mit Freunden, der bei Kontrolle auf Drittvariablen wie Erwerbsstatus und ethnische Zugehörigkeit verschwindet. Hier lassen sich die Unterschiede in der Kontakthäufigkeit also nicht durch die Armut erklären, sondern durch die Selektivität der Gruppe der Armen (über die die Autor*innen leider keine genauere Auskunft geben).

${ }^{4}$ Diese These ist hochinteressant, der Schluss von der Makroebene des Nationalstaats auf die Mesoebene sozialer Beziehungsnetzwerke der Akteur*innen scheint jedoch etwas gewagt. Kulturelle Unterschiede zwischen den untersuchten Ländern (z. B. Grad der sozialen Anerkennung bzw. Stigmatisierung von Armen), die ebenfalls Einfluss auf die Genese sozialer Beziehungen haben könnten, werden in dieser Analyse ausgeblendet.
} 
ähnlicher Lage - insbesondere dann, wenn die Betroffenen aus der Sphäre des Erwerbslebens ausgeschlossen sind (Domínguez \& Watkins, 2003). So stellen Böhnke und Link (2017) in einer Längsschnittanalyse auf Basis von SOEP-Daten fest, dass andauernde relative Einkommensarmut einen negativen Einfluss auf die Zahl erwerbstätiger - also statushöherer - Freund*innen im Netzwerk hat, während die Beziehungen zu Familienmitgliedern erhalten bleiben. Im Ergebnis entsteht der Eindruck kleinerer und homogenerer Familiennetzwerke.

Bei genauerem Hinsehen muss jedoch festgestellt werden, dass sich die Auswirkungen der Armut auf die Netzwerkgröße und -zusammensetzung nicht generalisieren lassen. Promberger et al. (2018) fordern daher, eine „Binnendifferenzierung" (ebd., S. 341) der Gruppe der Armen vorzunehmen, um Aufschluss über „außergewöhnliche Handlungspotentiale, Bewältigungsmuster und -verläufe in problematischen sozialen Situationen und Kontexten“ (ebd.) zu erlangen ${ }^{5}$. Zuletzt betonte Keck (2021), dass unterschiedliche Erscheinungsformen von Armut sowohl im Lebensverlauf als auch in Abhängigkeit der sozio-biographischen Positionierung auftreten. Viele qualitative Studien zur Wahrnehmung und Bewältigung von Erwerbslosigkeit, Prekarität und Armut kommen zu dem Ergebnis, dass es keine klassischen Armutsnetzwerke gibt, sondern eine Vielfalt an Handlungsstrategien, die jeweils in unterschiedlichen Beziehungskontexten zur Anwendung kommen (Klärner \& Knabe, 2016; Marquardsen, 2012; z. B. Marquardsen \& Röbenack, 2010; Weißmann, 2016). Die Autor*innen dieser Studien finden neben kleineren und homogeneren Netzwerken auch solche, die infolge von Prekarität oder Erwerbslosigkeit neu strukturiert wurden und eine soziale Teilhabe auf mehreren Dimensionen ermöglichen. Nicht immer lassen sich diese Dimensionen mit den Kategorien einer erwerbsarbeitszentrierten Perspektive erfassen, etwa dann nicht, wenn die Neupositionierung im Netzwerk um ein ehrenamtliches Engagement oder um eine ebenso sinnstiftende wie alltagsstrukturierende Tätigkeit im Familien- oder Nachbarschaftskontext herum gestaltet wird.

\subsubsection{Einflüsse auf die Netzwerkqualität und -ressourcen}

Studien zum Zusammenhang zwischen sozialen Netzwerken und sozialer Ungleichheit kommen zu dem Ergebnis, dass auch Netzwerkressourcen ungleich verteilt sind - Menschen mit geringerem ökonomischen und kulturellen Kapital

\footnotetext{
${ }^{5}$ Zur analytischen Vorgehensweise und zum Mehrwert von intra-Gruppenvergleichen für die Ungleichheitsforschung verweisen die Autor*innen auf Solga et al. (2013).
} 
verfügen in der Regel auch über weniger unterstützende und ressourcenreiche soziale Beziehungen (Böhnke \& Link, 2018; Dahl et al., 2008; Diewald, 1991; Diewald \& Lüdicke, 2007; Hollstein, 2018; Letki \& Mierina, 2015; Phan et al., 2009; Weyers et al., 2008). Mewes (2010) spitzt diese Feststellung auf die These einer „doppelten Exklusion“ (ebd., S. 208) zu. Demzufolge führt eine niedrige sozioökonomische Position (geringe Bildung, niedriger beruflicher Status) nicht nur zu einem erhöhten „Risiko der Desintegration in den Arbeitsmarkt und den damit verbundenen typischen Folgen (z. B. Armut, soziale Deprivation)“ (ebd.), sondern auch zu einer verminderten Leistungsfähigkeit sozialer Netzwerke. Netzwerke kompensieren andere Formen sozialer Ungleichheiten also nicht unbedingt. Sie sind häufig eher Teil der Akkumulation von Benachteiligungen (ebd. S. 205 ff.).

Darüber hinaus zeigen sich auch Einflüsse von Armut auf die Beziehungsintensität. So wird die Kontakthäufigkeit mit Personen jenseits der engsten Beziehungen mit andauernder Armut geringer (Böhnke \& Link, 2017; Cornwell \& Behler, 2015). Als Ursache dafür lassen sich die im Abschnitt zur Netzwerkgröße benannten Mechanismen anführen: formaler Ausschluss, Scham infolge von Stigmatisierung und Diskriminierung, nicht erfüllbare Reziprozitätsnormen, ein Mangel an Gelegenheitsstrukturen sowie fehlende Kapazitäten und Ressourcen zur Pflege der Beziehungen im Alltag.

\subsection{Wie wirken soziale Netzwerke auf Armut?}

Nicht nur die Armut beeinflusst das Netzwerk, auch über das Netzwerk können Mechanismen wirken, die die Armutssituation verändern. Zunächst wird aufgezeigt, wie soziale Netzwerke zur Entstehung, Reproduktion und Verschärfung von Armut beitragen können. Im zweiten Teil wird dann die entgegengesetzte Wirkrichtung betrachtet, der Erklärungsbeitrag sozialer Netzwerke für die Verringerung oder Überwindung von Armut.

\subsubsection{Entstehung und Reproduktion von Armut}

Im Wesentlichen lassen sich vier Wirkweisen sozialer Netzwerke auf die Entstehung und Reproduktion unterscheiden:

1. Negative Ties, also konflikthafte Beziehungen oder solche mit negativen Auswirkungen auf Ego können die Armutssituation verstärken (z. B. durch die 
Bindung von Kräften an die Sorge für Angehörige) oder Auswege aus der Armut versperren (Adebahr, 2020; Cattell, 2001; Keim-Klärner, 2020; Krause et al., 2008; Labianca \& Brass, 2006; Offer \& Fischer, 2018).

2. Abgrenzung nach oben: Objektive Benachteiligung kann zur Identifikation mit ebenfalls benachteiligten Peers führen, die sich in einer Abgrenzung von privilegierten Milieus äußert. Eventuell doch vorhandene Aufstiegschancen werden dann bewusst verneint (Willis, 1979).

3. Anschluss halten und Abgrenzung nach unten: Statusunsicherheiten führen dazu, dass davon Betroffene sehr viele Ressourcen und Energie aufbringen müssen, um die Zugehörigkeit zu ihrem Herkunftsmilieu zu verteidigen. In der Folge leben viele über ihre Verhältnisse und müssen diese Kosten auf der Hinterbühne ausgleichen, wo sich die Armutssituation verschärft (Ernährung, Wohnung, Gesundheit). Die Zugehörigkeit zu »besseren« sozialen Kreisen wird häufig durch eine Abgrenzung nach unten unterstrichen, welche den Potentialen für Solidarität und Unterstützung unter den Betroffenen entgegenläuft (Grimm, 2016; Hirseland \& Ramos Lobato, 2014; Knabe, Brandt et al., 2018; Knabe, Fischer et al., 2018; Kreher \& Matthäus, 2012; Schütt, 2014; Weißmann, 2016).

4. Schichtspezifische Beziehungsmuster machen Menschen mehr oder weniger abhängig von lokalen Gelegenheitsstrukturen. Die in unteren Schichten vermutlich häufiger vorzufindenden lagegebundenen Beziehungen werden prekär, sobald die mit ihnen verbundenen Kontexte (Arbeitsplatz, Vereinsheim, Dorffest) wegfallen oder die für die Nutzung dieser Gelegenheiten nötigen Ressourcen nicht mehr aufgebracht werden können (Allan, 1979; Hollstein, 2002, 2018).

Diese vier Mechanismen werden im Folgenden detailliert betrachtet.

\section{Negative Ties}

Ein Teil der armutsverstärkenden Wirkungen durch soziale Netzwerke lässt sich durch das Vorhandensein negativer Aspekte sozialer Beziehungen erklären. Zu unterscheiden sind dabei nach Adebahr (2020) Konflikte (Simmel, 1992, Kapitel IV: Der Streit), Abwertungen von Personen als ,negative Person“ (Labianca \& Brass, 2006, S. 597) sowie die Verhinderung des Zugangs zu Ressourcen durch Personen, die strukturell im Vorteil sind (Borgatti et al., 2018, 8 f.).

Cattell (2001) zeigt, dass die negativen Auswirkungen eines Mangels an Gelegenheitsstrukturen in der Nachbarschaft auf das Selbstvertrauen, das Wohlbefinden und die psychische Gesundheit dann besonders drastisch sind, wenn 
es zudem auch noch belastende Beziehungen in den Netzwerken gibt (Abhängigkeitsverhältnisse, häusliche Gewalt, sozialer Druck aus der Familie etc.). In diesen Fällen empfinden sich die Befragten als ausgeliefert. Sie geben ihre alltäglichen Bemühungen um Anschluss auf und neigen zu Hoffnungslosigkeit und Fatalismus.

Die Forschung zeigt jedoch, dass Beziehungen in der Regel komplex und selten eindeutig positiv oder negativ sind. So erfahren z. B. Alleinerziehende häufig Nachteile durch die mit der Sorge um die Kinder verbundenen Verpflichtungen. Dennoch würden sie ihre Kinder wohl kaum selbst als »negative « Beziehung charakterisieren (Keim-Klärner, 2020). Die hier vorgenommene Unterscheidung in armutsverstärkende und -hemmende Wirkungen ist daher eher analytisch zu verstehen.

Empirisch gemessen wurde der Zusammenhang zwischen dem sozioökonomischen Status und dem Vorhandensein negativer Aspekte sozialer Beziehungen in Netzwerke bislang selten (Adebahr, 2020). Die wenigen Analysen weisen jedoch darauf hin, dass vor allem Netzwerke von Personen mit sehr geringen materiellen Ressourcen oder mit Schulden besonders viele negative Ties aufweisen (Krause et al., 2008; Offer \& Fischer, 2018).

\section{Abgrenzung nach oben}

Paul Willis zeigt in seiner 1977 erschienenen ethnographischen Studie „Learning to Labour", wie weiße Jungen aus der englischen Arbeiterklasse ihre eigenen Benachteiligungen ausgerechnet dadurch reproduzieren, dass sie eine »Gegenschulkultur « entwickeln (Willis, 1979). In ihrer oppositionellen Haltung gegenüber der Schule sabotieren und boykottieren sie den Unterricht und damit auch ihre eigene Bildung. Ihre Form der Kritik an den herrschenden Verhältnissen und die damit verbundene Abgrenzung von den Werten und Normen des bürgerlichen Establishments resultiert aus einer starken Identifikation mit ihrem eigenen Milieu und reproduziert ihre soziale Benachteiligung: Sie werden als ungelernte Hilfsarbeiter in den Fabriken landen und keine sozialen Aufstiege erfahren (Willis, 1979).

Die Identifikation mit einer Netzwerkdomäne wie der Peergruppe und der sie umgebenden Kultur kann so gesehen dazu führen, dass Optionen jenseits dieser Domäne nicht mehr wahrgenommen werden (können). Auf diese Weise können Netzwerke zur (Re-)Produktion von Armut und Ungleichheit beitragen. Willis ist jedoch weit davon entfernt, den Jugendlichen selbst die »Schuld« an ihrer Situation zuzuschreiben. Er beschäftigt sich sehr ausführlich mit den Zusammenhängen zwischen den subjektiven Wahrnehmungen und kollektiven Verhaltensweisen der Jugendlichen und den objektiven gesellschaftlichen 
Ordnungs- und Ungleichheitsmustern, die diese hervorbringen. Er rekonstruiert akribisch, wie die im Alltag erfahrenen Benachteiligungen der Jugendlichen und ihrer Familien die Herausbildung der Gegenschulkultur befördern.

Doch damals wie heute gerät diese Form der Kontextualisierung von Erklärungen individueller Schicksale häufig in den Hintergrund. Was dann übrig bleibt, sind diskriminierende Klischees über angeblich leistungsunwillige Milieus, die den arbeitenden Schichten zur Last fallen und keinen eigenen »positiven« Beitrag leisten (Oschmiansky et al., 2003). Auf diese Weise wird die gesellschaftliche Verantwortung für die Armut geleugnet und auf die Einzelnen abgeladen. Sie sind dann ,ihrer sozialen Stellung nach nur arm [...] und weiter nichts“ (Simmel, 1992, S. 492). Im anglo-amerikanischen Raum wird in diesem Zusammenhang der Begriff der »Culture of Poverty« verwendet, der die Netzwerke von Menschen in Armut als »schädlich « diskreditiert, da diese negative Einflüsse, z. B. auf die Arbeitsmoral der Betroffenen, ausübten (Matthews \& Besemer, 2015). Empirische Belege für die Existenz solcher »Cultures of Poverty« oder »Cultures of Worklessness « gibt es jedoch genauso wenig (Macdonald et al., 2014) wie das typische Armutsnetzwerk, aus dem heraus sich das Phänomen hinreichend erklären ließe (Klärner \& Knabe, 2016; Wellman \& Potter, 1999) ${ }^{6}$.

\section{Anschluss halten und Abgrenzung nach unten}

Dennoch deuten die Kumulationseffekte ökonomischer, kultureller und sozialer Benachteiligungen darauf hin, dass Netzwerke zumindest nicht per se hilfreich sind bei der Bewältigung und Überwindung von Armut (Mewes, 2010). Der von Willis beobachtete von den »Armen« selbst ausgehende Abschluss nach oben wird in aktuellen Studien kaum mehr gefunden. Viele Studien - insbesondere in eher reichen Ländern mit wenigen Armen (Paugam, 2008) - weisen darauf hin, dass das Gegenteil der Fall sein könnte: Menschen in Armut orientieren sich eher an höheren Statuspositionen und grenzen sich von anderen Armen und nach unten ab. Häufig wird gezeigt, wie von Armut und Prekarität betroffene Personen infolge sozialer Abstiege und Statusunsicherheiten versuchen, ihre schwierige Lage zu verbergen, um ihre Zugehörigkeit zum Herkunftsmilieu (z. B. zu einem alten Freundes- und Kolleg*innenkreis) nicht zu gefährden (Grimm, 2016; Kreher \& Matthäus, 2012; Weißmann, 2016). Sowohl die materielle Knappheit als

\footnotetext{
${ }^{6}$ Zur Diskreditierung angeblich leistungsunwilliger Armer ließen sich auch zahlreiche öffentliche Äußerungen aus dem deutschsprachigen Raum anführen, die z. B. im Zuge der Einführung der Agenda 2010 sehr populär waren. Es ist aber unnötig, dieser hier zu wiederholen (siehe dazu auch: Lessenich 2003, 2012, S. 137 ff., bzw. Kronauer 2018; Stang 2018). Tiefergehend mit den Karrieren (pseudo-)wissenschaftlicher Semantiken sozialer Ungleichheit beschäftigt sich Otto (2019).
} 
auch ein makelbehafteter Status wie »erwerbslos « und die Gefahr einer Stigmatisierung erhöhen in diesen Fällen den individuellen Aufwand für die Pflege und Aufrechterhaltung von Beziehungen: Mit Goffman (1969) gesprochen muss daher auf der Hinterbühne gespart werden (Einrichtung der Wohnung, Heizkosten, Ernährung etc.), um in das eigene Erscheinungsbild auf der Vorderbühne (Kleidung, Statussymbole, Konsumverhalten) investieren zu können (Knabe, 2016; Knabe, Brandt et al., 2018).

Dieser Anpassungsdruck an statushöhere oder gesichertere Lagen kann zur Verstärkung von Armut und Prekarität beitragen: Viele leben über ihre Verhältnisse und laufen Gefahr, sich zu verschulden (Kutzner, 2016), sie sparen am Lebensnotwendigen (z. B. an Heizung und Ernährung) und riskieren so ihre Gesundheit (Knabe, Fischer et al., 2018; Krug et al., 2020) oder sie nehmen Hilfe nicht an, da ihre Strategie auffliegen könnte, wenn sie damit verbundene Reziprozitätserwartungen nicht erfüllen (ausführlich dazu in Abschnitt 3.1.1). Doch nicht nur ihre Verbindungen nach oben werden dadurch geschwächt - die gleichzeitige Abgrenzung nach unten (Grimm et al., 2013; Hirseland \& Ramos Lobato, 2014; Schütt, 2014) führt zu einer zusätzlichen Schwächung der Netzwerke, da Solidarisierungspotentiale und bonding social capital nicht ausgeschöpft werden.

\section{Schichtspezifische Beziehungsmuster}

Die oben zitierte Goffman'sche Begrifflichkeit der Vorder- und Hinterbühne (Goffman, 1969) lässt sich ideal auf die Analyse des Ringens um Kontrolle über die eigene Identität nach Harrison White anwenden (siehe Abschnitt 2.2.4). Akteur*innen haben die Möglichkeit, auf verschiedene Domänen zurückzugreifen, um sich in Szene zu setzen. Je mehr Domänen ihnen dafür zur Verfügung stehen, desto besser können sie diese strategisch im Rahmen von Kontrollversuchen einsetzen. Sie stellen die zur Selbstdarstellung besonders gut geeigneten Aktivitäten auf die Vorderbühne ihrer Außendarstellung und rücken die weniger repräsentativen Aspekte auf die Hinterbühne. Doch der Zugriff auf und die Bedeutung von Domänen scheint systematisch ungleich verteilt zu sein. Allan (1979) findet klassenspezifische Unterschiede bei der Bedeutung unterschiedlicher Orte für die Strukturierung von Beziehungen. Seine qualitative Studie zeigt, dass Arbeiter*innen eher kontextgebundene Freundschaften pflegen, die auf räumliche Nähe und zufällige Zusammentreffen in (semi-)öffentlichen Räumen wie Kneipe, Arbeit oder Nachbarschaft basieren. Demgegenüber würden Mittelschichtsangehörige eher de-kontextualisierte Freundschaften pflegen, die durch Verabredungen zu diversen gemeinsamen Gelegenheiten und Aktivitäten erhalten werden. Hollstein (2018) benennt in ihrer Rezeption dieser Studie materielle 
Ursachen, die zur Herausbildung dieser klassenspezifischen Beziehungsmuster beitragen:

\begin{abstract}
„Privatbesuche und -einladungen sind potenziell mit finanziellen Kosten wie auch mit möglichen Beeinträchtigungen des Selbstwertgefühls verbunden. Die Einladung selbst kann teuer sein. Möglicherweise schämt man sich, weil die Ausstattung der eigenen vier Wände als defizitär empfunden wird. Wenn man niemanden zu sich nach Hause mitnimmt, besitzt man eine größere Kontrolle über die Situation. “ (Hollstein, 2018, S. 201)
\end{abstract}

Wenn der eigene Haushalt also nicht geeignet ist, die eigene Identität im Rahmen von Kontrollversuchen in gutem Lichte dastehen zu lassen, fällt er als möglicher Ort der Begegnung weg. Dies ist bei Personen mit niedrigem Status häufiger der Fall als in statushöheren Lagen.

Bezogen auf die heutige Zeit unterscheidet Hollstein (2002) lage-gebundene und individualisierte Freundschaften. Sie nimmt an, dass sich diese ebenfalls systematisch nach vertikalen Schichtungsmerkmalen unterscheiden (Hollstein, 2018): Personen mit höherer Bildung verfügen demnach häufiger über individualisierte Freundschaften, während Menschen mit niedrigerer Bildung eher lage-gebundene Freundschaften pflegen. Eine quantitative Überprüfung für diese These steht jedoch bislang aus (ebd.). Wenn dem so sei, wäre der Zugriff auf Opportunitätsstrukturen in öffentlichen und teil-öffentlichen Räumen in niedrigen Lagen von größerer Bedeutung für das Ringen um Kontrolle der eigenen Identität als in höheren Lagen. Oder andersherum: Der Wegfall von Infrastrukturen (z. B. in ländlichen Regionen) träfe die ohnehin schlechter ausgestatten Gruppen stärker als andere (Klärner \& Knabe, 2019).

\title{
3.2.2 Verringerung und Überwindung von Armut
}

Auch die Mechanismen sozialer Netzwerke, die zu einer Verringerung oder Überwindung von Armut beitragen, lassen sich in vier Gruppen einordnen, wobei nur die zuerst genannte Gruppe ,soziale Unterstützung“ einen direkten Einfluss auf die Armut ausübt. Die folgenden drei Aspekte wirken eher indirekt als moderierende Effekte des Einflusses sozialer Einbindung auf die Chancen zur Verringerung der Armut:

1. Bedeutung sozialer Unterstützung für die Bewältigung von Armut: Armut kann durch soziale Unterstützung aus dem Netzwerk verringert oder sogar überwunden werden. Zu unterscheiden sind dabei unterschiedliche Arten und Quellen 
sozialer Unterstützung (Brandt, 2006; Diewald \& Sattler, 2010; Edin \& Lein, 1997; Granovetter, 1974; Nelson, 2000; Wellman \& Guila, 1999).

2. Aktivierung von Unterstützung: Die bloße Existenz von Bridging und Bonding Social Capital sagt noch nichts darüber aus, ob dieses tatsächlich abgerufen werden kann. Die Nutzbarmachung sozialen Kapitals erfordert Ressourcen und strategisches Geschick seitens der Unterstützungsempfänger*innen (Boon \& Farnsworth, 2011; Heflin et al., 2011; Nelson, 2000; Noordhoff, 2008; Ryser \& Halseth, 2011).

3. Prekäre Identitätsbehauptungen in und zwischen Domänen: Netzwerke sind eine wichtige Ressource zur Herstellung von Zugehörigkeit und Teilhabe. Diese steht im Falle von Armut und Prekarität infrage und muss über verschiedene Domänen hinweg verteidigt oder neu erschlossen werden (Grimm et al., 2013; Knabe, Brandt et al., 2018; Schütt, 2014).

4. Makrostrukturelle und kulturelle Einflüsse auf die Potentiale des Netzwerkhandelns: Aushandlungsprozesse in sozialen Netzwerken werden gerahmt durch kulturelle, normative und diskursive Deutungsmuster auf der Makroebene (z. B. Geschlechterrollen, Stigmatisierung, Sozialstaatliche Regulation), die potentiell mögliche Handlungsweisen in Netzwerken beschränken oder sogar spezifische Formen des Handelns in Netzwerken hervorbringen (Dieckhoff \& Gash, 2015; Hirseland \& Ramos Lobato, 2014; Krug et al., 2019; Motakef, 2019).

Diese vier Einflüsse werden im Folgenden ausführlich dargestellt.

1. Bedeutung sozialer Unterstützung für die Bewältigung von Armut

Der empirisch feststellbare Mangel an sozialer Unterstützung in den Netzwerken von Armut betroffener Menschen sollte nicht über den besonderen Bedarf und die hohe Bedeutung sozialer Unterstützung für die alltägliche Bewältigung und Verringerung von Armut hinwegtäuschen (Heflin et al., 2011; Nelson, 2000). In Anbetracht knapper Ressourcen und damit verbundener Notlagen wird Unterstützung durch Familienmitglieder, Institutionen und Freund*innen wichtiger für die Alltagsbewältigung (Edin \& Lein, 1997; Hill \& Kauff, 2001).

Differenziert werden können neben unterschiedlichen Formen sozialer Unterstützung, wie Rückhalt, Geselligkeit, Hilfe, Information, materielle Unterstützung usw. (Diewald \& Sattler, 2010), auch unterschiedliche Quellen sozialer Unterstützung, wie wohlfahrtsstaatliche Institutionen oder nachbarschaftliche, familiäre und freundschaftliche Beziehungen (Wellman \& Guila, 1999). Diese Typen von Akteur*innen stellen verschiedene Arten von Unterstützung bereit, die für von 
Armut betroffene Personen mehr oder weniger leicht zugänglich sind - beispielsweise abhängig von der sozial-räumlichen Distanz zwischen Alter und Ego (ebd. - siehe folgender Abschnitt).

In der Netzwerkforschung wird allgemein davon ausgegangen, dass sich die Unterstützung je nach Beziehungsstärke unterscheidet. Bonding Social Capital, welches über eher starke Beziehungen zu Personen in ähnlicher Lage vermittelt wird, bietet z. B. Zugang zu einfachen Arbeitsgelegenheiten, zu praktischer, motivationaler und emotionaler Unterstützung, die für die alltägliche Bewältigung von Armutssituationen von großer Bedeutung ist (Cattell, 2001; Domínguez \& Watkins, 2003). Allerdings sind die Ressourcen in sehr dichten und homogenen Netzwerken häufig redundant (ebd.). Eher über schwache Beziehungen zugängliches Bridging Social Capital eröffnet darüber hinausgehende Perspektiven durch die Bereitstellung von Informationen und Ressourcen jenseits des engsten Freundes- und Familienkreises (Brandt, 2006; Granovetter, 1973; Macdonald et al., 2014; Matthews \& Besemer, 2015).

\section{Aktivierung von Unterstützung}

Das bloße Vorhandensein von Bonding und Bridging Social Capital im Netzwerk allein bedeutet nicht unbedingt, dass die darin enthaltenen Ressourcen tatsächlich nutzbar gemacht werden können (Boon \& Farnsworth, 2011). Je nach Art der benötigten Unterstützung und Besonderheit der Unterstützungsgeber*innen müssen Akteur*innen unterschiedliche Strategien zur Anwendung bringen, um die Unterstützung abrufen zu können (Heflin et al., 2011).

Der Zugang zu Unterstützung ist von individuellen Kompetenzen abhängig und erfordert sehr viel Feingefühl und Geschick bei der Pflege sozialer Beziehungen. Nelson (2000) zeigt dies in ihrer qualitativen Studie am Beispiel der von Armut betroffenen Mütter, die zunächst einmal einschätzen müssen, wer von den Personen in ihrem Umfeld in der Lage ist, welche Art von Unterstützung zu leisten. Um die Unterstützung dann tatsächlich zu erhalten und mittelfristig abzusichern, müssen sie strategisch mit diesen Personen interagieren und die Beziehungen zu den Unterstützungsgeber*innen aktiv pflegen. In manchen Fällen ist es sogar ratsam, bestimmte Unterstützungsleistungen nicht anzunehmen, z. B., wenn die vorschnelle Annahme von Unterstützung die Beziehung gefährdet, da die erwartete Gegenleistung dafür nicht erbracht werden kann (Problem der Reziprozität, siehe 3.1.1). Weitere Gründe, Unterstützung im Netzwerk nicht anzunehmen, sind fehlendes Vertrauen in die Interaktionspartner*innen, der Anspruch an die Wahrung der eigenen Ehre (Freunde nicht ausbeuten wollen), der Wunsch nach Unabhängigkeit und Selbstbestimmung sowie die Vermeidung 
einer Außenwahrnehmung der eigenen Person als bedürftig (Noordhoff, 2008, S. 150).

Sowohl die strategische Zurückhaltung bei der Inanspruchnahme von Unterstützung als auch der Mangel an Unterstützung im erweiterten Netzwerk kann dazu führen, dass die engsten, meist familiären Beziehungen überlastet werden und den Bedarf an Unterstützung nicht mehr decken können (Jürgens, 2010; Ryser \& Halseth, 2011). Zusammenfassend muss daher festgestellt werden, dass soziale Unterstützung zwar ungemein hilfreich für die Bewältigung von Armut sein kann, jedoch nur selten in ausreichendem Maße zur Verfügung steht oder nutzbar gemacht werden kann.

\section{Prekäre Identitätsbehauptungen in und zwischen Domänen}

Insbesondere qualitative Studien weisen darauf hin, dass die Bedeutung von Netzwerken für die Bewältigung von Armut weit über den Zugang zu Unterstützung hinausgeht. Netzwerke sind eine Ressource zur Generierung sozialer Teilhabe und Zugehörigkeit. Sie bilden den mesostrukturellen Rahmen und damit auch den Möglichkeitshorizont des Bewältigungshandelns. Je sensibler die Analysen für die Bedeutung sozialer Beziehungen dabei sind, desto deutlicher wird, dass die (Wieder-)Herstellung von Zugehörigkeit auf der Möglichkeit beruht, sich Zugänge zu unterscheidbaren sozialen Kontexten und Domänen zu erhalten bzw. zu erarbeiten und Anerkennung von einer in die andere Domäne zu transferieren.

Wie bereits im Abschnitt 2.1.3 des Theorieteils erwähnt, identifizieren Grimm et al. (2013) in der qualitativen Panelstudie »Armutsdynamik und Arbeitsmarkt« (152 Befragte, 5 Wellen, 2007-2011) eine wachsende Zahl an Personen, die dauerhaft zwischen Hilfebezug und prekärer Beschäftigung wechseln und sich daher in einer »Zwischenzone « zwischen Erwerbslosigkeit und gesicherter Beschäftigung befinden (54\% der Befragten). Die Autor*innen rekonstruieren die Herausbildung eines „Zwischenzonenbewusstseins“ (ebd. S. 259) in dieser Gruppe, das sich einstellt, sobald die Befragten nicht mehr daran glauben, jemals wieder eine gesicherte Beschäftigung zu finden. Sie passen sich an die Situation an und versuchen, eine bestmögliche Absicherung auf diesem Niveau zu erreichen (Fixkosten reduzieren, für »Durststrecken« sparen, immer wieder nach neuen Beschäftigungen suchen). Die relative Stabilisierung in der Zwischenzone erfordert enorme Leistungen im Alltag der Befragten. Sie grenzen sich daher mit einem gewissen Stolz nach unten ab, von den Arbeitslosen, die diese Leistungen angeblich nicht erbringen. Vor welchem sozialen Hintergrund dieser Status einer positiv gelesenen Zwischenzonenidentität verteidigt wird, bleibt in dieser Studie jedoch etwas unklar - insbesondere in Anbetracht der Feststellung, dass mit 
zunehmender Aufenthaltsdauer in der Zwischenzone Beziehungen und Domänen (Hobbys, Freizeitaktivitäten) wegfallen und als weniger wichtig eingestuft werden, um die Verlusterfahrung auf diese Weise abzumildern.

Weiterführende Hinweise auf das Netzwerkhandeln in der Zwischenzone liefert die Arbeit von Schütt (2014), die ebenfalls mit Daten aus dem Projekt „Armutsdynamik und Arbeitsmarkt" arbeitet. Demzufolge versuchen Personen in prekären Lebenslagen, unterschiedliche Quellen sozialer Unterstützung miteinander zu kombinieren, um die größtmögliche Absicherung ihrer Situation zu erreichen. Dabei wird besonderes Augenmerk auf Beziehungen und Domänen gelegt, die zur Absicherung der Situation beitragen. Die bei Schütt analysierten Fallverläufe zeigen, dass „bisher stabile Beziehungen [...] im Hilfebezug verfestigt und gepflegt werden“" (ebd. S. 204), während eher destabilisierend wirkende Beziehungen gemieden oder abgebrochen werden (ebd. S. 204 ff.). Im Wesentlichen basiert die Absicherung in der Zwischenzone auf zwei Säulen. Eine Säule ist der Bezug von Transferleistungen, der jedoch an die Domänen des SGB-II-Regimes gekoppelt ist (Jobcenter, Maßnahmen, formalisierte Prozesse und Kontrollmechanismen), die ein „Bedrohungs- und Fremdbestimmtheitsgefühl“ (ebd., S. 240) aufkommen lassen. Um diese Abhängigkeiten zu verringern und selbstbestimmte Aktivitäten ausüben zu können, greifen die Befragten daher auf prekäre Beschäftigungen zurück. Jedoch kann auch in dieser Sphäre Autonomie nur befristet erreicht, nicht jedoch dauerhaft abgesichert werden. So sehen sich die Befragten ,in ihrer Wahrnehmung zwei Systemen gegenüber, die Sicherheit und Selbstbestimmung versprechen“ (ebd., S. 238), doch „,beide halten dieses Versprechen nicht" (ebd.). Keine der beiden Sphären lässt sich vollständig durch die andere kompensieren. So liegt es am individuellen Geschick der Betroffenen, das Verhältnis zwischen den beiden Sphären so auszubalancieren und durch alternative Domänen sozialer Teilhabe zu ergänzen (Schütt benennt die Arbeitslosenselbsthilfe und Ehrenamt), dass sie der Erfüllung ihrer Ansprüche an Teilhabe, Autonomie und Absicherung so nahe wie möglich kommen.

Bislang beschäftigen sich nur wenige Studien explizit aus einer Netzwerkperspektive mit den Anpassungsleistungen von Menschen in Armut und Prekarität. Knabe, Brandt et al. (2018) fragen (u. a. unter Verwendung eines Teils der in dieser Arbeit verwendeten Daten), wie Anerkennungsdefizite vor dem Hintergrund eines unfreiwilligen Ausschlusses aus der Sphäre der Erwerbsarbeit kompensiert werden, und rücken dabei den Mechanismus des Netdom Switchings in den Mittelpunkt ihrer Betrachtung. Sie stellen fest, dass Arbeitslose und prekär Beschäftigte versuchen, Anerkennung in alternativen Domänen ihrer Netzwerke zu generieren. Dies gelingt ihnen jedoch in Abhängigkeit von den sie umgebenden Gelegenheitsund Anerkennungsstrukturen nur teilweise (durch bewusstes Raushalten der mit 
der Erwerbslosigkeit verbunden Probleme aus dem Freundeskreis) oder gar nicht (sie sind dem Vorwurf unzureichenden Bemühens ausgesetzt). Die Autor*innen schließen daraus, dass die für die Generierung von Anerkennung nutzbaren Domänen an den Rändern der Erwerbsgesellschaft ,seltener und unbeständiger“ (Knabe, Brandt et al., 2018, S. 190) sind.

4. Makrostrukturelle und kulturelle Einflüsse auf die Potentiale des Netzwerkhandelns

Motakef (2019) geht genauer auf die Aushandlung sozialer Anerkennung auf der Mikroebene der Paarbeziehung und deren makrostrukturelle Prägungen ein. Sie zeigt, wie die in der Arbeitswelt beeinträchtigte Anerkennung teilweise durch Liebes-Anerkennung und eine Anpassung partnerschaftlicher Arrangements bei der Aufteilung von Care- und Erwerbsarbeit kompensiert werden kann. Inwieweit Paare jedoch in der Lage sind, alternative Handlungsweisen zu etablieren, wird in entscheidendem Maße von gesellschaftlichen Normen und Werten wie der Akzeptanz alternativer Geschlechterrollen geprägt und ist abhängig von deren Verbindlichkeit innerhalb der Partnerschaft (ebd., siehe auch: Motakef \& Wimbauer, 2019; Wimbauer \& Motakef, 2018, 2020).

Ebenfalls auf der makrostrukturellen Ebene lassen sich die in jüngerer Zeit häufiger analysierten Auswirkungen von Stigmatisierung auf die Wahrnehmung und Bewältigung von Armut und Erwerbslosigkeit zuordnen (Gurr et al., 2018; Gurr \& Lang, 2018; Knabe, Fischer et al., 2018; Lang \& Gross, 2019; Linden et al., 2018). Hirseland und Ramos Lobato (2014) zeigen, dass gesellschaftliche Klischeebilder über faule Arbeitslose ein Handeln auf der Individualebene herausfordern. Erwerbslos zu sein bedeutet eine Infragestellung der gesellschaftlichen Stellung, welche die Betroffenen auf drei Weisen zu entkräften versuchen:

1. Compliance: Die Demonstration ,der »richtigen inneren Haltung « auf Ebene der Selbstpräsentation“ (ebd. S. 193, Hervorhebung im Original), indem die Betroffenen zeigen, dass sie die an sie als Erwerbslose gestellten Erwartungen voll und ganz zu erfüllen bereit sind.

2. Solidarisierung mit der öffentlichen Meinung: „die sich [...] in der Übernahme und Reproduktion jener »herumgeisternden Klischees« und in einer affirmativen Haltung gegenüber den damit verknüpften Aktivierungsimperativen und Disziplinarmaßnahmen zeigt“ (ebd. S. 193, Hervorhebung im Original). Hier beteiligen sich die Betroffenen also an der Abwertung von Arbeitslosen und stimmen der Sanktionierung und Verdächtigung dieser Gruppe prinzipiell zu. Damit ordnen sie sich diskursiv der Seite der Nicht-Erwerbslosen zu und grenzen sich von den anderen Erwerbslosen ab. 
3. „Positionierung des je eigenen Falls als Ausnahme vom angeblichen Regelfall bzw. des Typus des »arbeitsunwilligen Hilfeempfängers" " (ebd. S. 194, Hervorhebung im Original): Die Betroffenen betrachten sich als Einzelfälle, die außerhalb des ansonsten gültigen Klischeebildes stehen.

Diese Strategien legen die Schlussfolgerung nahe, dass eine Solidarisierung oder die Herausbildung eines Klassenbewusstseins, wie es vielleicht im Fall der Zwischenzone beobachtet wurde (siehe oben) ${ }^{7}$, bei Erwerbslosen nicht zu erwarten ist.

Der Einfluss wohlfahrtsstaatlicher und normativer Kontexte auf der Makroebene bestätigt sich bei der Betrachtung international vergleichender Studien zur sozialen Teilhabe von Menschen in relativer Einkommensarmut. Dieckhoff und Gash (2015) zeigen in einer Analyse auf Basis der EU-SILC-Daten, dass der negative Zusammenhang zwischen Erwerbslosigkeit und sozialer Partizipation (Vereinsmitgliedschaft, Häufigkeit außerhäuslicher Aktivitäten, Kontakthäufigkeit mit Freund*innen, Verfügbarkeit sozialer Unterstützung) vor allem dort besonders drastisch ausfällt, wo die Erwerbslosen einem besonders hohen Armutsrisiko ausgesetzt sind und dass die Arbeitslosen dort, wo sich die Politik eher am Ideal der Umverteilung ausrichtet, in geringerer Weise von einem Mangel an sozialer Partizipation betroffen sind. Diese Beobachtung führen sie zu folgender Schlussfolgerung:

"This suggests that the normative environment has a structuring effect on social participation, which confirms our hypothesis that societal attitudes can encourage or dissuade the social participation of outsider groups." (Dieckhoff \& Gash, 2015, S. 82)

Zusammenfassend lässt sich feststellen, dass Netzwerke eine wichtige Ressource im Rahmen der Armutsbewältigung darstellen. Die Verfügbarkeit dieser Ressource ist jedoch ungleich verteilt und wird durch kulturelle, normative und politische Rahmenbedingungen reguliert.

\footnotetext{
${ }^{7}$ Auch hier ist zu fragen, ob es sich beim Zwischenzonenbewusstsein (Grimm et al. 2013, S. 259) tatsächlich um ein Klassenbewusstsein im Marx'schen Sinne handelt, welches die Zugehörigkeit zu einer sozialen Gruppe und deren innere Verbundenheit mit einschließt, oder ob damit eher der (durchaus berechtigte) Stolz auf die individuell erbrachte Anpassungsleistung gemeint ist.
} 


\subsection{Typologien von Netzwerken und Beziehungen}

Keck (2021) zufolge ist die generalisierte Betrachtung einer homogenen Klassenlage der Armen nicht dazu geeignet, das Phänomen hinreichend zu erfassen. Ebensowenig dürfe man aber auf die gegenteilige Annahme einer gänzlich individualisierten Armut verfallen, da es sowohl innerhalb als auch zwischen den verschiedenen Armutsgruppen strukturelle Gemeinsamkeiten gibt. Eine Typisierung von Armut liegt daher nahe, die der Komplexität des Phänomens gerecht wird, ohne dabei ins Beliebige abzudriften. Typologien der Bewältigung von Armut in sozialen Netzwerken zeigen in zugespitzter Weise, dass Menschen in Abhängigkeit der sie umgebenden Strukturen unterschiedlich mit dem Problem materieller Knappheit umgehen. Es lassen sich fünf Arten der Typisierung von Netzwerken unterscheiden:

1. Typisierung von Netzwerken unter Verwendung standardisierter Netzwerkmaße (Keim, 2011; Klärner \& Knabe, 2019; Wellman \& Potter, 1999),

2. Typisierung nach den für das Bewältigungshandeln relevanten Netzwerkressourcen und Beziehungsarten (Cattell, 2001; Domínguez \& Watkins, 2003; Marquardsen, 2012),

3. Typisierung domänenspezifischer Beziehungskulturen (Allan, 1979; Hollstein, 2002; Nelson, 2000),

4. Typisierung alltäglicher Wahrnehmung und des Handelns in Netzwerken (Grimm, 2016; Jahoda et al., 1975 [1933]; Weißmann, 2016).

Im Folgenden sollen Beispiele für jede dieser fünf Gruppen aufgezeigt werden, um besser einschätzen zu können, auf welche Teilbereiche des Zusammenhangs zwischen Armut und Netzwerk bereits erstellte Typologien blicken und welche Differenzierungen sie anhand welcher Kriterien vornehmen.

\subsubsection{Typisierung auf Basis standardisierter Netzwerkmaße}

Unabhängig von einer konkret inhaltlichen Fragestellung versuchen Wellman und Potter (1999), die Strukturen egozentrierter Netzwerke explorativ zu untersuchen und systematisch voneinander zu unterscheiden. Sie entwickeln aus einer Faktorenanalyse $^{8}$ von 33 standardisiert erhobenen egozentrierten Netzwerken, die im

8 ,promax factor analysis“ S. 57 - ähnliche Ergebnisse haben die Autor*innen mit hierarchical cluster analysis und multidimensional scaling erzielt. 
Kontext einer größeren Erhebung zu den sozialen Beziehungen von 845 Bewohner*innen eines Stadtteils in Toronto generiert wurden, vier Dimensionen zur Unterscheidung von Netzwerkstrukturen und -zusammensetzungen:

1. Das Verhältnis von verwandten zu nicht-verwandten Alteri

2. die Kontakthäufigkeit und Erreichbarkeit der Alteri

3. die Reichweite des Netzwerkes (Größe und Heterogenität) und

4. die Intensität (,Intimacy“ ebd. S. 67) der Beziehungen.

Diese vier Dimensionen zur Unterscheidung von Netzwerken lassen sich bei einer binären Codierung (high/low) auf 16 verschiedene Weisen miteinander kombinieren. 13 dieser 16 Kombinationen sind tatsächlich im Sample zu finden (ebd., S. 71). Der am häufigsten vorkommende Netzwerktyp (14\% der Fälle) beschreibt Netzwerke mit einer hohen Beziehungsintensität und -reichweite sowie einer hohen Kontakthäufigkeit und einem geringen Anteil an Verwandten. Wellman und Potter (1999) schließen aus ihrer Analyse, dass es sich bei persönlichen Netzwerken um komplexe Strukturen handelt, die sich nicht ohne Weiteres generalisieren lassen. Wer nach dem einen typischen Armuts-Netzwerk sucht, würde demnach scheitern.

Allerdings stößt die Aussagekraft rein quantifizierender Analysen von Netzwerkmaßen schnell an ihre Grenzen. Über die Feststellung der Unterschiedlichkeit hinaus bleibt zu fragen, was genau wir eigentlich messen, wenn wir Netzwerke erfassen. In Bezug auf die Studie von Wellman und Potter (1999) könnte man auch fragen, warum die Autor*innen ausgerechnet die oben genannten Maße zur Differenzierung von Netzwerken heranziehen und nicht etwa die Netzwerkdichte oder die Modularität verwenden. Keim (2011) erarbeitet daher eine Netzwerktypologie auf Basis eines methodenintegrativen Forschungsprozesses. Sie typisiert zunächst die Fertilitätsintentionen von jungen Erwachsenen qualitativ und betrachtet dann die Verteilung der standardisierten Maße zu den egozentrierten Netzwerken innerhalb der Typen. Sie stellt fest, dass sich die Verteilungen der Netzwerkmaße systematisch zwischen den qualitativen Typen unterscheiden und integriert so ihre quantitativen $\mathrm{Maße}$ in ihre qualitative Typologie (z. B. Family-Centered, Polarized, Non-Supportive).

Im Bereich der Armutsforschung verfolgen Klärner und Knabe (2019) einen ähnlichen Ansatz unter Verwendung der in dieser Arbeit tiefergehend analysierten 
Daten ${ }^{9}$. In einem ersten Schritt vergleichen sie die Mittelwerte der standardisierten Maße zu den egozentrierten Netzwerken der Befragten mit Wohnsitz in der Stadt $(n=33)$, mit jenen, die im ländlichen Raum $(n=16)$ wohnen. Dabei stellen sie fest, dass die im ländlichen Raum erhobenen Netzwerke kleiner und enger verbunden sind, einen höheren Anteil an familiären Alteri sowie einen geringeren Anteil an unterstützenden Beziehungen aufweisen. Im zweiten Schritt typisieren sie die Bewältigungsstrategien der 16 im ländlichen Raum wohnenden Befragten entlang der Dimensionen Zukunftsperspektiven (,future prospects“ ebd., S. 458) und Selbstwirksamkeit (,self-efficacy“, ebd.). Die Zukunftsperspektive schwankt dabei zwischen Veränderung (,,change“, ebd.) und Stagnation, die Selbstwirksamkeit der Befragten wurde zwischen den Polen selbstbestimmt (,self-determined“, ebd.) und fremdbestimmt (,other-directed“, ebd.) angesiedelt:

- Zukunftsperspektive-Veränderung: Personen, die davon ausgehen, dass in Zukunft Veränderungen in ihrem Leben eintreten, gehen demnach davon aus, dass sie diese selbst herbeiführen werden (Typ: „precarious but resilient" ebd. - selbstbestimmt), oder hoffen auf Veränderung durch Impulse von außen (Typ: ,dependent but hopeful“" ebd. - fremdbestimmt).

- Zukunftsperspektive-Stagnation: Diejenigen, die keine Veränderung erwarten, haben sich entweder so an die Armutssituation angepasst, dass sie einigermaßen gut damit leben können und gegen weitere Rückschläge abgesichert sind (Typ: „adaption and maintenance “ ebd. - selbstbestimmt), oder sie sind unzufrieden mit der Situation und fühlen sich dauerhaft benachteiligt und um ihre Lebenschancen betrogen (Typ: „disappointment and resignation“ ebd. - fremdbestimmt).

Mit Blick auf die egozentrierten Netzwerke der Befragten zeigt sich, dass sich die Beziehungsstrukturen zwischen den einzelnen Typen stark voneinander unterscheiden (ebd., S. 466): Akteur*innen mit hoher Selbstwirksamkeit haben unabhängig von ihrer Zukunftsperspektive heterogenere und funktional differenziertere Netzwerkstrukturen, während Akteur*innen mit geringer Selbstwirksamkeit über eher kleinere und eng verbundene Netzwerke mit wenig brückenbildendem sozialen Kapital verfügen.

\footnotetext{
${ }^{9}$ Analysiert wurden 49 der 57 egozentrierten Netzwerke aus der Studie Aspekte der Armut in Mecklenburg-Vorpummern (Klärner et al. 2015). Die darin enthaltene Kontrastgruppe der 8 Studierenden im Sample wurde in der Analyse von Klärner und Knabe (2019) aus der Analyse ausgeschlossen. Befragt wurden Menschen aus städtischen und ländlichen Regionen, die unterhalb der Einkommensarmutsgrenze von $60 \%$ des Landesmedians in MecklenburgVorpommern leben.
} 
Die Arbeiten von Keim (2011) und Klärner und Knabe (2019) weisen auf einen Zusammenhang zwischen Netzwerkstruktur und Handeln hin: Standardisierte Netzwerkmaße lassen sich nur im Vergleich zwischen kategorial differenzierten Gruppen (Stadt/Land) oder im Zusammenhang mit den sie umgebenden Sinnstrukturen interpretieren. Die Bedeutung unterschiedlicher Netzwerkstrukturen lässt sich nicht aus den Maßzahlen ableiten, sondern muss aus der Akteur*innenperspektive heraus rekonstruiert werden.

Offen bleibt am Ende jedoch die Frage nach dem Verhältnis von Handeln und Struktur bei der Herausbildung unterschiedlicher Netzwerktypen im Längsschnitt. Bilden sich bestimmte Typen von Netzwerken infolge von Egos Handeln heraus oder erzeugt das Netzwerk die spezifische Handlungsweise von Ego?

"Initially, we can therefore only confirm that there is a correspondence between certain network structures and certain fertility-related attitudes and behaviours, but not say anything about the direction of the effect - unless we take a closer look at network dynamics and processes of social influence." (Keim, 2011, S. 217)

Einige qualitative Studien nehmen daher eine biographisch-rekonstruktive Perspektive ein (Marquardsen, 2012 - siehe Abschnitt 3.3.2) oder arbeiten mit qualitativen Wiederholungsbefragungen (Grimm et al., 2013; Grimm, 2016 - siehe Abschnitte 3.2.2 und 3.3.3). Geeignete quantitative Datensätze zur Beantwortung der Frage des Zusammenwirkens von Netzwerkstruktur und Handeln im Längsschnitt fehlen bislang jedoch weitgehend (Reis et al., 2020).

\subsubsection{Typisierung von Netzwerkressourcen und Beziehungen}

Cattel (2001) untersucht die Wechselwirkungen zwischen sozialem Netzwerk und den sozial-räumlichen Kontexten der Nachbarschaften von Armut betroffener Menschen in zwei Londoner Stadtteilen auf Basis einer qualitativen Studie mit etwa 100 Befragten. Sie identifiziert fünf Typen sozialer Netzwerke von Menschen in Armut (socially excluded, homogeneous, traditional, heterogeneous, solidarity), die sich im Hinblick auf die Ressourcen unterscheiden, die im Kontext der Bewältigungsstrategien der Befragten relevant werden. Eher homogene Netzwerkstrukturen bieten Gelegenheit zu wechselseitiger praktischer Unterstützung und Rückhalt in der Armutssituation (socially excluded, homogeneous). Aufgrund der Ähnlichkeit der Alteri sind die Netzwerkressourcen jedoch eher redundant. Heterogenere Netzwerke eröffnen den Zugang zu einer breiteren Palette an 
Ressourcen und Gelegenheiten, die zur Verringerung der Armut beitragen können (heterogeneous). Einen Mischtyp bilden die „Networks of solidarity“ (ebd., S. 1512), bestehend aus Personen, die sich stark in solidarischen, eher homogenen Peer-Gruppen organisieren (Familien, Vereine), die jedoch auch Zugänge in darüberhinausgehende Domänen bieten.

Domínguez und Watkins (2003) unterscheiden in ihrer qualitativen Studie die Netzwerke geringverdienender alleinerziehender Mütter mit Migrationshintergrund in Boston nach dominanten Beziehungsarten in familiäre, freundschaftliche und institutionelle Netzwerke. Sie betonen, dass Unterstützung aus allen Netzwerktypen generiert werden kann, wobei die Art der Unterstützung in eher homogenen Netzwerken einseitiger ist und weniger Perspektiven eröffnet als in heterogeneren Netzwerken.

Ganz ähnlich unterscheidet Marquardsen (2012) drei Arten sozialer Netzwerke $^{10}$ von Erwerbsarbeitslosen nach den „,bei der Bewältigung dominanten Beziehungsarten“ (ebd. S. 250): Familie, Freundschaft und semi-professionelle Beziehungen. Er stellt fest, dass sich die Netzwerktypen systematisch entlang der beiden Dimensionen „Handlungsorientierungen“ (ebd.) und „Veränderungen im sozialen Netzwerk" (ebd.) seit dem Eintreten der Erwerbslosigkeit verteilen:

1. Handlungsorientierung: Die Inhaber*innen von Freundschaftsnetzwerken und semi-professionellen Netzwerken streben eher nach Autonomie, während Personen mit Familiennetzwerken eher nach Sicherheit suchen.

2. Veränderungen im sozialen Netzwerk: Kontinuitäten über die Zeit vor und seit Eintreten der Erwerbslosigkeit lassen sich vor allem für die Freundschaftsnetzwerke feststellen und für solche Familiennetzwerke, die auch vor Eintreten der Erwerbslosigkeit schon stark abgeschottet gegenüber externen Beziehungen waren. Brüche in den Netzwerken finden sich bei denen, die nach Eintreten der Erwerbslosigkeit semi-professionelle Beziehungen aufgebaut haben, sowie in auf die Familie reduzierten Netzwerken, bei denen es zum Verlust externer Beziehungen infolge der Erwerbslosigkeit kam (ebd. S. 250 ff.).

Die Typologie zeigt, dass es einem Teil der Befragten gelingt, Netzwerke zu erhalten oder sogar neue Domänen zu erschließen, während der Verlust der

\footnotetext{
${ }^{10}$ Er analysiert die Netzwerke von 27 überwiegend erwerbslosen Personen auf Basis problemzentrierter Interviews und vorstrukturierten Netzwerkkarten, auf denen Ego in der Mitte eingetragen ist, umgeben von konzentrischen Kreisen, die die dort durch die Befragten einzuzeichnenden Alteri als „sehr unterstützend“ bis ,ein wenig unterstützend“ klassifizieren (ebd. S. 95).
} 
Erwerbsarbeit andere auf ohnehin eher schwache Netzwerke zurückwirft oder zur Erosion von Beziehungen jenseits der Familie führt.

\subsubsection{Typisierung domänenspezifischer Beziehungskulturen}

Auf die Typisierung von Beziehungskulturen wurde im Abschnitt 3.2.1 bereits unter Verweis auf die kontextgebundenen, bzw. de-kontextualisierten Beziehungen bei Allan (1979) bzw. die lage-gebundenen und individualisierten Freundschaften bei Hollstein (2002) eingegangen. Auf Basis einer qualitativen Studie mit 39 alleinerziehenden Müttern mit geringem Einkommen aus dem ländlichen Raum bietet die Studie von Nelson (2000) ein weiteres Beispiel für die Typisierung von »Beziehungskulturen«. Sie stellt fest, dass Reziprozitätsnormen in Abhängigkeit von den Eigenschaften der Interaktionspartner*innen unterschiedlich ausgelegt werden. Mit Personen in gleicher Lage pflegen die Befragten möglichst ausbalancierte Austauschbeziehungen (Typ 1) - was die Mütter bekommen und was sie dafür zurückgeben, ist etwa gleichwertig. Bei bessergestellten Personen entfällt die Norm der Gleichwertigkeit der ausgetauschten Ressourcen (Typ 2) - eine Befragte gießt bspw. die Blumen im Haus der sie weit umfänglicher unterstützenden Eltern, wenn diese verreist sind. Insbesondere jene Mütter ohne stärkere Beziehungen zu Freund*innen oder Verwandten weiten die Reziprozitätsnorm über die konkreten Interaktionspartner*innen hinaus aus (Typ 3) - da jede*r mal großzügig zu irgendwem sein sollte, ist es legitim, Unterstützung anzunehmen, ohne dafür in dem konkreten Kontext eine Gegenleistung zu erbringen (generalisiertes Vertrauen nach Putnam 2001).

\subsubsection{Typisierung des Handelns in Netzwerken}

In dieser Arbeit wird die Bedeutung von Netzwerken für die Herstellung von Handlungsfähigkeit unter der Bedingung von Armut analysiert. Daher sind vor allem jene Typologien von besonderem Interesse, die die Wahrnehmung und Bewältigung von Armut und Prekarität danach differenzieren, wie Zugehörigkeit und Teilhabe konkret unter der Bedingung von Armut im Netzwerk hergestellt werden.

In der Studie von Jahoda et al. (1975 [1933]) steht der Umgang mit den Folgen des Wegfalls der Domäne des Arbeitsplatzes und der damit verbundenen materiellen Knappheit im Mittelpunkt der Betrachtung. Die Autor*innen 
treffen in ihrer Typologie eine grundsätzliche Unterscheidung, die in fast allen Studien zur Wahrnehmung und Bewältigung von Armut wiederzufinden ist: Die Marienthaler werden zwischen den beiden Polen „gebrochen“ (ebd., S. 71) und „ungebrochen“ (ebd.) typisiert. Manchen Befragten gelingt es, sich der Situation anzupassen (Typ: ungebrochen-resigniert, ebd. S. 70) oder sogar Perspektiven zu ihrer Überwindung zu entwickeln (Typ: ungebrochen, ebd. S. 71), andere verlieren jede Lebensfreude und Hoffnung (Typ: gebrochen-verzweifelt, ebd.) oder geben sich und die alltäglichen Reproduktionstätigkeiten nahezu ganz auf (Typ: gebrochen-apathisch, ebd. S. 71 f.).

Ganz im Einklang mit der Literatur über den Zusammenhang zwischen Netzwerkstruktur und Bewältigungschancen sind sich Jahoda et al. (1975 [1933]) am Ende jedoch nicht sicher, ob es sich hierbei wirklich um eine Typologie mehr oder weniger resilienter Charaktere oder um ein Phasenmodell des stufenweisen Niedergangs handelt (102 ff.). Der Faktor Zeit scheint neben der subjektiven und der strukturellen Perspektive auf jeden Fall eine wichtige Rolle zu spielen, mit der sich die empirische Forschung zur Wahrnehmung und Bewältigung von Armut bis heute schwertut.

Auch bei Sammet (2014) taucht die Unterscheidung zwischen Personen auf, die der Erwerbslosigkeit eigene Handlungsweisen entgegenzusetzen (»ungebrochen «, hier: intentionale Prozessstruktur) haben, und solchen, die sich einer als gegebenen wahrgenommenen Situation eher unterordnen (»gebrochen«, hier: konditionale Prozessstruktur). Sie typisiert die Weltsichten von 41 Erwerbslosen unter Rückgriff auf die Konzepte aus Durkheims (1983) Selbstmordstudie. Ein Teil der Befragten wähnt sich in einer sinnhaft geordneten Welt (Eunomia), die anderen empfinden ihre Welt dagegen als überreguliert und fatalistisch oder als ungeordnet und anomisch. Aus allen drei Zuständen heraus kann Handlungsfähigkeit hergestellt werden (intentionale Prozessstruktur): als „trotziges Aufbegehren“ gegen die Überregulierung (Fatalismus), als „Herstellung der (guten) Ordnung“ (Eunomia) oder als „Bewältigung von Kontingenz“ (Anomie). Doch nicht bei allen Befragten lassen sich diese Formen von Agency tatsächlich beobachten (konditionale Prozessstruktur): So wird in einigen Fällen die Kontingenz einfach hingenommen (Anomie), vor der eigenen Machtlosigkeit „resigniert“ (ebd. S. 84 - Fatalismus) oder die als sinnhaft empfundene Ordnung akzeptiert (Eunomia). Der letzte Punkt weist darauf hin, dass fehlende Agency nicht immer ein Problem darstellen muss, sondern auch aus dem Gefühl des Aufgehobenseins in einer Struktur entstehen kann. Über die für diese Arbeit sehr interessante Rekonstruktion und Typisierung von Weltsichten hinaus bietet die Studie jedoch keine systematischen Analysen zu deren Entstehungskontexten in den Netzwerken der Befragten. 
Grimm (2016) ${ }^{11}$ fokussiert dagegen stärker auf die individuellen Anpassungsleistungen und das Netzwerkhandeln unter prekären Bedingungen. Sie analysiert die Wahrnehmung prekär gewordener Statuszuordnungen von Menschen in unsicheren Beschäftigungsverhältnissen (Zwischenzone zwischen Hilfebezug und Erwerbstätigkeit, siehe auch: Grimm et al., 2013 bzw. Abschnitt 3.2). Die Befragten erleben Statusinkonsistenz ihrer Typologie (ebd. S. 390) folgend entweder als Bedrohung (Typ 1), als Herausforderung (Typ 2), als Bürde (Typ 3) oder als normale Passage ihres Lebensverlaufs (Typ 4). Die von Grimm beschriebenen Einflüsse auf diese unterschiedliche Wahrnehmung lassen sich in vier Dimensionen zusammenfassen (ebd. S. 390 f.):

1. Grad der Verinnerlichung des Leistungsideals (Eigenverantwortlichkeit vs. Glück/Pech)

2. Zukunftsorientierung (langfristige Planung vs. Gegenwartsorientierung),

3. Verknüpfung von Status und Erwerbstätigkeit (Verschmelzung vs. Kompensation im Privaten) und

4. familiäre biographische Prägung (Statuskonsistenz vs. Statusinkonsistenz in der Herkunftsfamilie).

Theoretisch gehaltvoll mit Blick auf die Agency in sozialen Beziehungsnetzwerken ist Grimms Rückgriff auf den Begriff der Statusinkonsistenz bei der Erarbeitung der Typologie. Sie rekonstruiert Statusinkonsistenz als subjektive Wahrnehmung, die relational zu gesellschaftlich erzeugten Normalitätserwartungen und den individuellen Erfahrungen mit Diskontinuitäten im Lebensverlauf erzeugt und in den Dimensionen der Typologie sichtbar gemacht wird. Netzwerktheoretisch gedeutet ließe sich diese Perspektive auf der 4. und 5. Dimension von Identitäten nach White verorten (siehe Abschnitt 2.2): Die subjektive Wahrnehmung von Status(in)konsistenz erschiene dann als Identität, die aus der Interpretation der Selbstbeobachtung von Akteur*innen zwischen Netzwerkdomänen in der Zeit entsteht.

Auch Weißmann (2016) nimmt mit ihrer Arbeit zur Herstellung von Zugehörigkeit unter der Bedingung von Langzeiterwerbslosigkeit eine relationale Perspektive ein. Sie zeigt, dass der von der gesellschaftlichen Normalitätserwartung abweichende Status »erwerbslos « die soziale Zugehörigkeit der Betroffenen infrage stellt, wodurch ein aktives Handeln der Betroffenen herausgefordert wird.

\footnotetext{
${ }^{11}$ Das Sample besteht aus 38 Personen aus der qualitativen Längsschnittstudie „Armutsdynamik und Arbeitsmarkt", die Statusinkonsistenzen im Beobachtungszeitraum durchlebt haben.
} 
Zugehörigkeit muss unter dieser Bedingung neu konstruiert und produziert werden. Dies tun die Akteur*innen, indem sie biographisch erlernte Muster der Herstellung von Zugehörigkeit weiterentwickeln und in unterschiedlichen sozialen Sphären zur Anwendung bringen. Einige der Befragten schaffen es auf diese Weise (z. B. durch die Ausübung erwerbsähnlicher Tätigkeiten in der Familie oder im Rahmen eines Engagements), eine gewisse „Normalisierung“ (ebd. S. 236) ihrer Situation zu erreichen, durch die die diskreditierende Wirkung der Erwerbslosigkeit verringert wird (Typ 1). Anderen gelingt die Herstellung von Zugehörigkeit über solche normalisierenden Strategien unter Betonung eigener Anstrengungen weniger gut („Modus des Prozessiertwerdens“ ebd., S. 238, Typ 2) oder nur in einer bestimmten Sphäre, in der sie sich als Expert*innen (Typ 3: Statusnivellierung) oder Aktivist*innen (Typ 4: Selbstermächtigung) etablieren, ohne dass ihnen diese Rolle über den konkreten Kontext hinaus soziale Anerkennung einbringt. Damit zeigt Weißmann, dass es möglich ist, die negativen Auswirkungen von Erwerbslosigkeit unter Rückgriff auf Netzwerkdomänen zu verringern oder die Erwerbstätigkeit sogar durch alternative Tätigkeiten zu ersetzen. Die Zugehörigkeit kann jedoch erst dann vollständig wiederhergestellt werden, wenn es gelingt, erfolgreiche Kontrollversuche in einem Teilbereich des Netzwerks über mehrere Domänen hinweg zu etablieren.

\subsection{Zusammenfassung, Desiderate und Fragestellung der Arbeit}

In der Literaturstudie zu den Wechselwirkungen zwischen Armut und sozialen Netzwerken wurden drei Teilbereiche untersucht. Im ersten Teil wurden Auswirkungen von Armut auf soziale Netzwerke diskutiert. Es zeigt sich, dass Armut in Wohlstandsgesellschaften häufig zu einer Verkleinerung sozialer Netzwerke und zu einer Homogenisierung der Beziehungsarten führt. Oft werden Netzwerkdynamiken beschrieben, die bei andauernder Armut zu einer Reduktion auf ein familiäres Kernnetzwerk führen. Ursächlich dafür ist u. a. der Mangel an kulturellen und materiellen Ressourcen, die für die Pflege (reziproker) Austauschverhältnisse benötigt werden. Infolgedessen sind die Potentiale sozialer Netzwerke im Hinblick auf die Verringerung und Überwindung von Armut begrenzt. Mewes (2010) spricht in diesem Zusammenhang von einer doppelten Exklusion bzw. der Kumulation von Benachteiligungen.

Im zweiten Teil wurden die Erklärungsbeiträge sozialer Netzwerke auf die Entstehung und Reproduktion von Armut sowie die Verringerung und Überwindung von Armut betrachtet. Armutsverstärkend können negative Auswirkungen 
sozialer Beziehungen (z. B. Stress aufgrund von sozialem Druck, Sorgearbeit, Kontrolle), Abgrenzungsbedürfnisse nach oben (z. B. symbolische Abgrenzung von normativen Anforderungen privilegierter Schichten) und unten (Abwertung anderer Armer «) sowie die stärkere Abhängigkeit niedrigerer sozialer Schichten von lokalen und öffentlich zugänglichen Gelegenheitsstrukturen wirken. So macht etwa der beengte Wohnraum die Pflege sozialer Beziehungen stärker abhängig von der Verfügbarkeit an Treffpunkten in öffentlich Räumen.

Armutsverringernd wirken soziale Netzwerke als wichtigste Quelle sozialer Unterstützung in Anbetracht materieller Knappheit und als Arenen der Herstellung sozialer Zugehörigkeit. Hierbei fällt auf, dass der Zugang zu potentiell im Netzwerk verfügbaren Ressourcen auch vom individuellen Geschick der Akteur*innen bei der Pflege und Strukturierung ihrer Beziehungen abhängt. Allerdings kann dieses Geschick nicht beliebig eingesetzt werden, sondern nur im Kontext makrostruktureller Rahmenbedingungen (Stigmatisierung, Anerkennungsordnungen, Diskurse und Regelungen zu Armut und Erwerbslosigkeit etc.). Die Entscheidung für eine bestimmte Bewältigungsstrategie wird darüber hinaus nicht isoliert in einem Teilbereich des Netzwerks getroffen, sondern unter Berücksichtigung der sozialen und gesellschaftlichen Einbettung insgesamt. So können etwa besonders riskante Strategien (wie die Annahme einer unsicheren Arbeitsstelle) nur dann gewählt werden, wenn das Risiko dieser Handlung in einer anderen Domäne abgefedert werden kann (z. B. durch sozialen Rückhalt in der Familie oder die Verfügbarkeit institutioneller Unterstützung bei der Kinderbetreuung).

Bis hierhin zeigte das Literaturstudium, dass Netzwerke selten einheitlich strukturiert sind. Daher wurden im dritten Teil Möglichkeiten der Typisierung von Armut im Kontext sozialer Beziehungsnetzwerke erörtert, mittels derer sich die Heterogenität des Bewältigungshandelns in sozialen Netzwerken unter der Bedingung von Armut abbilden lässt. Netzwerke lassen sich nach statistischen Kennziffern typisieren (3.3.1), nach den Ressourcen, die sie den Akteur*innen bereitstellen, sowie nach den dominanten Beziehungsarten (3.3.2). Darüber hinaus gibt es Typisierungen von Beziehungskulturen im Netzwerk (z. B. nach spezifischen Reziprozitätsnormen - 3.3.3) und Typologien des Netzwerkhandelns, bei denen unterschiedliche Arten der Herstellung von Zugehörigkeit herausgearbeitet werden (3.3.4). Aus der Betrachtung der Typologien lässt sich lernen, dass Netzwerkstrukturen und -zusammensetzungen nur im Kontext der Handlungs- und Deutungsweisen der Akteur*innen sinnvoll interpretiert werden können.

Von besonderer Bedeutung im Kontext der Fragestellung dieser Arbeit sind die zuletzt genannten Typisierungen des Netzwerkhandelns. Hier fällt auf, dass sich die Unterscheidung zwischen »gebrochenen« und »ungebrochen« Armen 
(Jahoda et al., 1975 [1933]) wie ein roter Faden durch die Analysen zieht. Sie kennt zahlreiche Variationen, die in der Regel auf der Unterscheidung zwischen eher von den Akteur*innen selbst ausgehenden und eher von außen gesteuerten Wahrnehmungs- und Bewältigungsweisen basieren: self-determined/otherdirected (Klärner \& Knabe, 2019), direkte vs. vermittelte Zugehörigkeitsherstellung (Weißmann, 2016), Autonomie vs. Sicherheit (Marquardsen, 2012), intentionale vs. konditionale Prozessstruktur (Sammet, 2014) usw.

Der im Theorieteil dieser Arbeit eingeführte Agency-Begriff wird in den betrachteten Studien nur selten verwendet. Dennoch ist die Handlungs- und Gestaltungsfähigkeit der Akteur*innen häufiger Bestandteil der Analysen. Ein Teil der Arbeiten untersucht diese hinsichtlich der Anpassungsfähigkeit an eine gegebene Situation, der andere Teil fokussiert eher auf die Fähigkeit, konkrete Handlungsziele zu erreichen. Bezüglich der Anpassungsfähigkeit lassen sich drei Schwerpunkte ausmachen:

1. Bewältigung von Erwerbslosigkeit (Edin \& Lein, 1997; Jahoda et al., 1975 [1933]; Klärner \& Knabe, 2016; Marquardsen, 2012; Sammet, 2014; Schütt, 2014)

2. Bewältigung von Prekarität (Gefken, 2018; Grimm et al., 2013; Grimm, 2016; Knabe, Brandt et al., 2018)

3. Bewältigung von Armut (Cattell, 2001; Domínguez \& Watkins, 2003; Klärner \& Knabe, 2019)

Bewältigung wird in diesen Kontexten als die Suche nach einem möglichen Umgang mit einer als gegeben wahrgenommene Situation begriffen, wobei es nicht primär um die Überwindung des als problematisch wahrgenommenen Zustands geht. Agency als Fähigkeit zur Erreichung konkreter Handlungsziele wird darüber hinaus mit Blick auf folgende Themenbereiche in der Literatur behandelt:

1. Erwerbslosigkeit überwinden (Brandt, 2006; Krug et al., 2019),

2. Unterstützung rekrutieren (Nelson, 2000; Ryser \& Halseth, 2011)

3. Zugehörigkeit und Anerkennung herstellen (Knabe, Brandt et al., 2018; Kutzner, 2016; Motakef, 2019; Motakef \& Wimbauer, 2019; Weißmann, 2016),

4. Stigmatisierung vermeiden (Gurr \& Lang, 2018; Hirseland \& Ramos Lobato, 2014; Knabe, Fischer et al., 2018). 


\section{Kontextualität}

Gelegenheitsstrukturen, Positionen, Verhältnisse und Diskurse
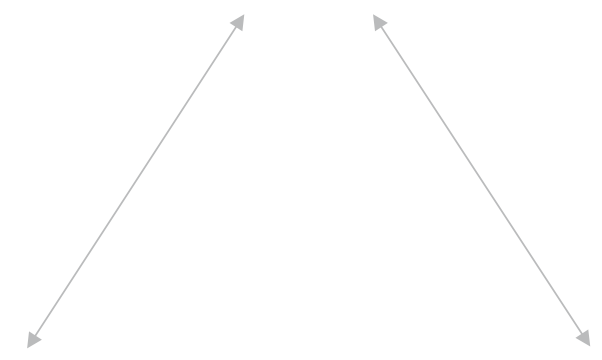

\section{Agency}

Subjektive Wahrnehmung der

Situation und Handlungsfähigkeit
Relationalität

Einbettung in Strukturen sozialer Netzwerke

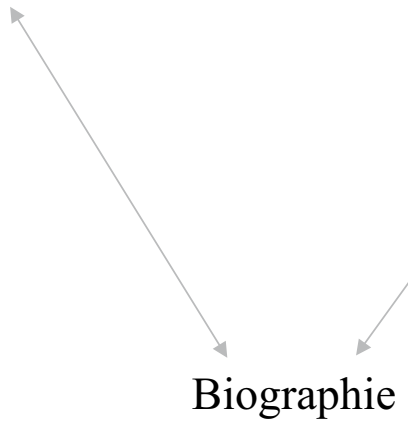

Erlebnisse von Selbstwirksamkeit, Einübung von Rollen und Identitäten

Abbildung 3.1 Mehrdimensionale Analyseperspektive

Bei näherem Hinsehen zeigt sich, dass die analytische Trennung zwischen Agency als Anpassungsfähigkeit und als Fähigkeit, Ziele zu erreichen, in der Praxis der Akteur*innen verschwimmt. So muss die Vermeidung von Stigmatisierung nicht unbedingt als Handlungsziel operationalisiert werden. Sie kann auch Teil einer Strategie der Anpassung an eine prekäre Lebenssituation und des Statuserhalts sein. Genauso kann eine Anpassungsstrategie an die Armut zu 
deren Überwindung beitragen (z. B. dann, wenn die Integration in eine alternative Domäne in einem auskömmlichen Erwerbsverhältnis mündet). Die Akteure sind häufig in beiden Feldern gleichzeitig aktiv: Solange die prekäre Situation nicht überwunden werden kann, sind sie darauf zurückgeworfen, das Beste daraus zu machen. Daher erscheint es nicht sinnvoll, Agency auf eine der beiden Perspektiven zu verkürzen.

Die Literaturrecherche betont die Notwendigkeit einer Mehrebenenbetrachtung der Wahrnehmung und Bewältigung von Armut, die in Abbildung 3.1 zusammengefasst ist. Die Agency (subjektive Ebene) steht in engem Zusammenhang mit der sozio-biographischen Prägung der Akteur*innen. Diese Prägung wird in dieser Arbeit als Spur von Ein- und Entbettungen in die Domänen sozialer Netzwerke im Zeitverlauf begriffen (Relationalität auf der Mesoebene sozialer Netzwerke). Das Netzwerkhandeln wird beeinflusst durch übergeordnete gesellschaftliche Diskurse und Rahmenbedingungen (Kontextualität auf der Makroebene).

Open Access Dieses Kapitel wird unter der Creative Commons Namensnennung 4.0 International Lizenz (http://creativecommons.org/licenses/by/4.0/deed.de) veröffentlicht, welche die Nutzung, Vervielfältigung, Bearbeitung, Verbreitung und Wiedergabe in jeglichem Medium und Format erlaubt, sofern Sie den/die ursprünglichen Autor(en) und die Quelle ordnungsgemäß nennen, einen Link zur Creative Commons Lizenz beifügen und angeben, ob Änderungen vorgenommen wurden.

Die in diesem Kapitel enthaltenen Bilder und sonstiges Drittmaterial unterliegen ebenfalls der genannten Creative Commons Lizenz, sofern sich aus der Abbildungslegende nichts anderes ergibt. Sofern das betreffende Material nicht unter der genannten Creative Commons Lizenz steht und die betreffende Handlung nicht nach gesetzlichen Vorschriften erlaubt ist, ist für die oben aufgeführten Weiterverwendungen des Materials die Einwilligung des jeweiligen Rechteinhabers einzuholen.

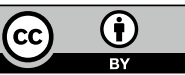

